\title{
Consumption of OLL1073R-1 Yogurt Improves Psychological Quality of Life in Women Healthcare Workers: A Randomized Controlled Trial
}

Tetsu Kinoshita ( $\nabla$ tetsu.prospective@gmail.com )

Ehime University

Koutatsu Maruyama

Ehime University

Keiko Suyama

Ehime University

Mariko Nishijima

Ehime University

Kimiko Akamatsu

Ehime University

Akiko Jogamoto

Ehime University

Kikumi Katakami

Ehime University

Isao Saito

Oita University

\section{Research Article}

Keywords: Yogurt, Lactobacillus delbrueckii ssp. bulgaricus OLL1073R-1, woman's health, quality of sleep, psychological quality of life

Posted Date: January 15th, 2021

DOl: https://doi.org/10.21203/rs.3.rs-137029/v1

License: (c) (i) This work is licensed under a Creative Commons Attribution 4.0 International License. Read Full License 


\section{Abstract}

Background To investigate the effects of yogurt consumption fermented with Lactobacillus delbrueckii ssp. bulgaricus OLL1073R-1 on improving mental quality of life of women healthcare workers, a randomized controlled trial was conducted. The 961 women (mainly nurses, aged 20-71 years) agreed to participate in this trial, and were randomly allocated to either the yogurt group $(n=479)$ or the control group ( $\mathrm{n}=482$ ). Participants of the yogurt group drank $112 \mathrm{~mL}$ of OLL1073R-1 yogurt for 16 weeks, meanwhile those of the control group did not consume the yogurt. All participants were prohibited from consuming other yogurt or fermented dairy products during the study period. The participants answered the Pittsburgh Sleep Quality Index (PSQI), Short Form-8 Health Survey (SF-8), and Gastrointestinal Symptom Rating Scale (GSRS) questionnaires at baseline and after 16 weeks.

Results The PSQI score showed significant improvement after the intake of yogurt $(p<0.01)$. SF-8 results showed significant intervention effects in the General Health and Vitality scores $(p=0.02$ and $p=0.01$, respectively). In other subscales of SF-8, we did not observe significant effects of the yogurt. In the GSRS, daily intake of the yogurt exerted a preventive effect on constipation $(p=0.03)$.

Conclusions Consumption of yogurt fermented with Lactobacillus delbrueckii ssp. bulgaricus OLL1073R1 enhances subjective psychological quality of life by improving quality of sleep and gastrointestinal condition among women healthcare workers.

\section{Background}

It has been established that yogurt, a fermented dairy product, has beneficial effects on certain gastrointestinal conditions including lactose intolerance, constipation, inflammatory bowel diseases, and Helicobacter pylori infection [1, 2]. Recent studies have investigated the role of yogurt in enhancing human immune function by changing the balance of the intestinal microbiota and stimulating the intestinal immune system via lactic acid-producing bacteria or substances produced by the bacteria $[3,4]$. Furthermore, some studies have attempted to demonstrate the beneficial effects of probiotics on mental health, i.e., ameliorating depression, anxiety, fatigue, and sleep disturbance [5-11]. However, the reported results have been inconsistent. Psychological disorders impair the social functions of individuals, reduce the production efficiency of workers, and cause comorbidity with physical disorders [12]. Therefore, the beneficial effects of probiotics with respect to improving mental health should be further investigated to reduce the burden of psychological disorders among the working population.

A previous study showed that yogurt fermented with Lactobacillus delbrueckii ssp. bulgaricus OLL1073R1 (OLL1073R-1), which produces a large amount of exopolysaccharide (EPS), reduced the risk of catching a cold in elderly subjects. Moreover, improvements in quality of life (QOL) in areas such as "Lacks general motivation", "Irritation", "Stress," and "Easily fatigued" were also observed in the study [13]. However, previous studies have not comprehensively evaluated the effect of yogurt on QOL-related overall health indices. Based on the previous results [13] and expected benefits of probiotics [1, 2], we hypothesized that 
consumption of OLL1073R-1 yogurt may exert beneficial effects on improving psychological QOL and QOL-related overall health among other populations.

We conducted the present randomized controlled trial to evaluate the effects of OLL1073R-1 yogurt on improving psychological QOL and QOL-related overall health among women healthcare workers. Women healthcare workers are constantly exposed to the risk of infection because they are in prolonged contact with patients suffering from infectious diseases. In addition, their level of psychological QOL may be lower than that associated with other occupations due to the irregular nature of shift work [14]. Many working women are also busy with childcare and housework, and thus especially in need of health support. For those reasons, we selected women healthcare workers as the participants in this trial.

\section{Results}

Table 1 shows the baseline characteristics of the participants. At baseline, there was no significant difference identified between the two groups for any of the indices.

Table 2 shows the scores of PSQI, SF-8, and GSRS at baseline and after 16 weeks in each group. Mean PSQI scores at baseline and after 16 weeks were 5.50 and 5.03 in the yogurt group, and 5.33 and 5.22 in the control group, respectively. The observed intervention effect was statistically significant $(p<0.01)$. For SF-8, the intervention effects were significant in the GH and VT scores $(p=0.02$ and $p=0.01$, respectively). The mean GH scores at baseline and after 16 weeks were 49.2 and 50.1 in the yogurt group, and 49.1 and 48.9 in the control group, respectively. The mean VT scores at baseline and after 16 weeks were 49.6 and 50.5 in the yogurt group, and 49.4 and 49.2 in the control group, respectively. We did not observe significant effects in other subscales, physical component summary, and MCS. The intervention effect in the GSRS score reached statistical significance for constipation $(p=0.03)$. Geometric mean constipation scores at baseline and after 16 weeks were 1.74 and 1.72 in the yoghurt group, and 1.76 and 1.84 in the control group, respectively. No statistically significant intervention effects were observed in the other subscales and total score.

Table 3 shows the correlation matrix between the changes in PSQI, GSRS, and QOL scores in the yogurt group. In the VT and MCS scores, a weak inverse correlation with PSQI was demonstrated $(r=-0.26,-0.22$, respectively). No correlation between the changes in PSQI and GSRS scores was observed $(r=0.08)$.

There were no serious side effects caused by the intake of yogurt [15].

\section{Discussion}

The primary outcome of this trial was to evaluate the preventive effects of yogurt fermented with $L$. bulgaricus OLL1073R-1 against influenza. Consumption was not associated with reduced influenza incidence but was associated with an increase in interferon gamma production [15]. This paper covers secondary outcomes of the same trial, not covered in the previous report: namely, sleep quality, subjective QOL, and gastrointestinal condition among women healthcare workers. Significant improvements in PSQI 
score, psychological QOL scores of the SF-8, and constipation score of the GSRS associated with yogurt intake were demonstrated.

A novel finding of the present study was improvement in the quality of sleep as a result of the consumption of OLL1073R-1 yogurt. This effect may have significantly improved the GH and VT scores of the SF-8. As shown in Table 3, significant correlations were observed between the PSQI score and VT score. Therefore, we suspect that the observed increases in the GH and VT scores reflected reduced fatigue, itself attributable to improved sleep quality. A recent, randomized, placebo-controlled trial demonstrated the anti-fatigue effect of OLL1073R-1 yogurt among healthy male volunteers suffering from summer heat fatigue [22]. With respect to the GSRS, the intervention effect on the constipation score was statistically significant. This result suggests that constipation was prevented rather than exacerbated by the daily intake of yogurt (constipation score: 1.74-1.72) in the yogurt group. In the control group, constipation worsened due to the restrictions on yogurt intake (1.76-1.84). The effect of probiotics on improving constipation has been documented in several previous clinical trials [23-26].

The mechanisms of yogurt fermented with L. bulgaricus OLL1073R-1 involved in the quality of sleep and fatigue are not fully understood. However, the EPS produced by OLL1073R-1 is considered one of its active ingredients. Several studies reported that purified polysaccharides exert an anti-fatigue effect [2733]. Mushroom polysaccharide, known for its antioxidant activity, free radical scavenging activity, immunomodulatory activity, and maintenance of normal liver function, is involved in the anti-fatigue effect [34]. OLL1073R-1 EPS may exhibit anti-fatigue effects via similar mechanisms. In addition, it contains immunostimulatory phosphopolysaccharides [35] and possesses antioxidant activity, which have also been reported in EPS produced from another lactic acid bacterium [36-39].

The strength of the present study lies in the use of a large-scale, randomized, controlled design. A previous study which evaluated the anti-fatigue effect of the yogurt was small-scale [22]. The present results can provide a new evidence in the field of nutritional psychology. Another strength is that this large-scale study was performed under stringent conditions (i.e., participants did not consume any other fermented dairy products during the 16-week trial). Despite this restriction, the withdrawal rate was $<3 \%$ $(n=25)$, and $>900$ participants completed the trial.

This study had several limitations. Firstly, participants were not blinded, which means placebo foods were not prepared for the control group. Since it was impossible to prepare a placebo yogurt with similar characteristics (i.e., appearance, taste, and flavor) and deliver the blinded yogurts (active or placebo) to each participant every week, we employed an open-label design. As a result, for some participants in the control group who were eager to be in the yogurt group, they might have some stress due to the complete yogurt restriction. On the other hand, the yogurt group participants would have been motivated to complete the trial. Consequently, the differences in the mood or stress levels between the groups might have affected the results. Secondly, since all participants could not consume any fermented dairy products except for the test food (for the yogurt group) by the rule of the study, some kind of selection bias might have been generated. Some workers refused to participate in informed consent sessions 
because of this limitation. To eliminate this effect, it may be desirable to select participants who do not have a habit of taking probiotics. Thirdly, all participants were women. Although equality of sexes is preferred to better understand the effects, many clinical studies have been conducted with female populations, making results more comparable.

\section{Conclusions}

The present study involving women healthcare workers showed the effects of OLL1073R-1 yogurt intake on improving quality of sleep, fatigue, sense of general health, and constipation. Our findings by this trial would provide new insights into the field of nutritional psychology and contribute to a better comprehension for the benefits of probiotics in our society. Further clinical trials, which employ appropriate sample sizes and randomized controlled trial design, are needed to validate the effects of yogurt or fermented dairy products.

\section{Methods}

\section{Study design and participants}

This trial was a randomized, controlled, and open-label study. The main objective of the trial was to examine the effects of OLL1073R-1 yogurt on the incidence of influenza among women healthcare workers [15]. This paper details the secondary, psychological health-related outcomes monitored in the same trial.

The main contents of methods of the present trial were explained in the previous report [15]. We estimated that a sample size of 600 participants in each group would be required to detect the effects of OLL1073R-1 yogurt on the incidence of influenza. Participants were recruited from 23 medical institutions in Ehime prefecture, Japan, from August to September, 2016. Women (aged $\geq 20$ years) who were currently employed as medical or welfare-related professionals at medical institutions in Ehime prefecture and could understand the study purpose were selected, with written informed consent. The exclusion criteria were as follows: (1) pregnancy; (2) contracting influenza during the period from July 2016 to the date of providing written informed consent; (3) allergic responses to dairy products; (4) lactose intolerance; (5) instructed to restrict calorie intake by a physician; (6) history of diseases involving the immune system (e.g., rheumatism, cancer, thyroid disorder, systemic lupus erythematosus, myasthenia gravis, Graves' disease, scleroderma); (7) participation in other clinical trials within the past 3 months; and (8) judged as unsuitable by the principal physician for other reasons. Of the 1,026 women who agreed to participate in this study, 20 infringed the exclusion criteria and 24 declined to participate after the agreement. Therefore, 982 women were examined at the screening session. By the day of the examination session, 20 women infringed the exclusion criteria and one declined participation. Consequently, 961 women (aged 20-71 years) were enrolled in the present study. 
All participants were instructed not to consume any yogurt or fermented dairy foods from the day of agreement to that of random assignment. A total of 961 women were assigned to either the yogurt group $(n=479)$ or the control group $(n=482)$ through block randomization within three strata: institutions of employment, age, and having a plan of influenza vaccination or not.

Participants in the yogurt group consumed the test yogurt daily for 16 weeks (from November 14, 2016 to March 5, 2017), whereas those in the control group did not consume yogurt during this period.

Furthermore, all participants were instructed not to consume any other yogurt or fermented dairy products throughout this trial. The participants answered self-administrated questionnaires at baseline and after 16 weeks. In addition, participants were provided with a "health notebook" to record changes in their lifestyles, intake of the test yogurt (for the yogurt group), and intake of any other yogurt or fermented dairy foods (for both groups) during the trial period. During the trial, four and 12 participants in the yogurt and control group declined participation, respectively. In the control group, two participants did not attend the examination session and one did not complete the self-administered questionnaires 16 weeks later. In addition, two and four participants in the yogurt group and control group, respectively, revealed that they were pregnant during the trial, thereby infringing the exclusion criteria. Figure 1 shows the sampling scheme throughout this study.

\section{Test yogurt}

The test food was "Meiji Probio Yogurt R-1" drink type (Meiji Co., Ltd., Tokyo, Japan), which is currently available on the market. This yogurt is manufactured using two lactic acid bacterial species, namely $L$. bulgaricus OLL1073R-1 and a strain of Streptococcus thermophilus, originally isolated from traditional Bulgarian yogurt. One bottle contains $112 \mathrm{~mL}$ of drinkable yogurt and provides $76 \mathrm{kcals}, 13.9 \mathrm{~g}$ carbohydrate, $0.67 \mathrm{~g}$ fat, $3.6 \mathrm{~g}$ protein, and $\geq 1.12 \times 10^{9} \mathrm{CFU}$ (colony forming units) of L. bulgaricus and S. thermophilus.

\section{Outcome measurement}

The participants completed the self-administrated questionnaires discussed below at baseline and after 16 weeks.

\section{Quality of sleep}

The Pittsburgh Sleep Quality Index (PSQI) was used as an index of subjective sleep condition [16], as it is established that dairy products exert beneficial effects on relaxation and quality of sleep [17]. The questionnaire is self-administered, and the participants were asked to answer the questions according to their quality of sleep (i.e., onset, duration, efficiency, difficulty, medication, and daytime sleepiness) during the previous 1 month. Each answer is converted into the score and the total score reflects the comprehensive sleep condition. Higher scores represent worse condition.

\section{Subjective QOL}


The eight-item Short Form Health Survey (SF-8) was used to evaluate subjective QOL [18]. The effect of OLL1073R-1 on improving mood status have been previously reported [13]. Therefore, we examined its role in improving psychiatric QOL. The SF-8 is a generic questionnaire widely used to compare the impact of different medical conditions, and as an outcome measure of different therapeutic interventions. The questionnaire is self-administered, and the participants were asked to complete eight questions according to their experiences during the previous 1 month. The questions were divided into eight subscales and two dimensions that described the overall health status. The eight subscales were physical functioning $(\mathrm{PF})$, role physical (RP), bodily pain (BP), general health $(\mathrm{GH})$, vitality $(\mathrm{VT})$, social functioning (SF), role emotional (RE), and mental health (MH). The two dimensions are physical component summary (PCS) and mental component summary (MCS), were calculated based on the scores of the eight subscales using specific standardized algorithms. The scores of each scale ranged from 0 to 100 , with high scores representing better QOL.

\section{Gastrointestinal condition}

The Gastrointestinal Symptom Rating Scale (GSRS) was used as an index of gastrointestinal condition $[19,20]$, as it is established that probiotics improve gastrointestinal symptoms, such as constipation [2]. The GSRS consists of 15 questions; the participants answered all questions according to their gastrointestinal condition during the previous 1 week. We evaluated the total score and five subscale scores (i.e., reflux of acid, gastric pain, indigestion, diarrhea, and constipation). The scores of the total and each subscale ranged from 1 to 7 , with higher scores representing worse condition.

\section{Statistical analysis}

All statistical analyses were performed using based on intention-to-treat analysis in SAS version 9.4 (SAS Institute Inc., Cary, NC, USA). Differences in the means of the indices between the two groups at baseline and after 16 weeks were analyzed using an unpaired t-test. The effects of the intervention on the scores of the PSQI, SF-8, and GSRS were analyzed through two-way analysis of variance with repeated measures. Owing to the non-normal distribution of the GSRS score, geometric means were calculated and used for analysis. In case of participant withdrawal from the study, the values for PSQI, SF-8, and GSRS obtained at baseline were used as those after 16 weeks. In the yogurt group, the correlation between the changes in QOL scores and changes in PSQI and GSRS scores were analyzed using Spearman's rank correlation analysis. For the incidence of adverse events in both groups, the rates were analyzed in 10 categories using the chi-squared test. In all analyses, $p<0.05$ (two-sided) denoted statistical significance.

\section{Declarations}

\section{Ethics statement}

The present study was approved by the Institutional Review Board (IRB) of Ehime University Hospital and the IRB of the Meiji Corporation. Written informed consent was provided by all participants. The reporting of this trial follows the recommendations of the CONSORT (Consolidated Standards of Reporting Trials) 2010 statement [21]. This trial was registered at the University Medical Information Network Clinical Trial registry as UMIN-RCT Identifier UMIN000023932 (10/09/2016). In addition, the study was conducted in 
accordance with the protocol and Japanese ethical guidelines for medical and health research involving human subjects.

\section{Acknowledgement}

The authors thank to all participants, doctors, and chief nurses of 23 medical institutions for cooperation in experiments. In addition, we would like to thank Uni-edit (https://uni-edit.net/) for editing and proofreading this manuscript. This study was supported by funding from the Meiji Corporation (Tokyo, Japan).

\section{Author contributions}

The author's responsibilities were as follows: TK, KM, and IS conceived and designed the study; TK, KS, $\mathrm{MN}, \mathrm{KA}, \mathrm{AJ}$, and KK led and conducted research; KM and IS analyzed data; TK, KM, an IS wrote the paper; and all authors read and approved the final manuscript.

\section{Competing interests and Funding}

Tetsu Kinoshita (first author) is the head of the Institute of Community Life Sciences Co., Ltd (Matsuyama, Japan). This study was funded by the Meiji Corporation (Tokyo, Japan) based on the contract agreement between the Meiji Corporation and the Institute of Community Life Sciences. The Meiji Corporation had no input into the data analysis and interpretation. The other authors had no personal or financial conflict of interest.

\section{Data availability}

The datasets generated during the current study and analyses are available from the corresponding author on reasonable request.

\section{References}

1. Adolfsson O, Meydani SN, Russell RM. Yogurt and gut function. Am J Clin Nutr. 2004;80:245-56

2. George KR, Patra JK, Gouda S, Park Y, Shin HS, Das G. Benefaction of probiotics for human health: A review. J Food Drug Anal. 2018;26:927-39.

3. Meydani SN, Ha WK. Immunologic effects of yogurt. Am J Clin Nutr. 2000;71:861-72.

4. Hooper LV, Littman DR, Macpherson AJ. Interactions between the microbiota and the immune system. Science 2012;336:1268-73.

5. Slykerman RF, Hood F, Wickens K, Thompson JMD, Barthow C, Murphy R, et al. ; Probiotic in Pregnancy Study Group. Effect of Lactobacillus rhamnosus HN001 in pregnancy on postpartum symptoms of depression and anxiety: a randomised double-blind placebo-controlled trial. EBioMedicine 2017;24:159-65. 
6. Allen AP, Hutch W, Borre YE, Kennedy PJ, Temko A, Boylan G, et al. Bifidobacterium longum 1714 as a translational psychobiotic: modulation of stress, electrophysiology and neurocognition in healthy volunteers. Transl Psychiatry. 2016;6:e939.

7. Östlund-Lagerström, L. Kihlgren A, Repsilber D, Björkstén B, Brummer RJ, Schoultz I. Probiotic administration among free-living older adults: a double blinded, randomized, placebo-controlled clinical trial. Nutr J. 2016;15:80.

8. Takada M, Nishida K, Kataoka-Kato A, Gondo Y, Ishikawa H, Suda K, et al. Probiotic Lactobacillus casei strain Shirota relieves stress-associated symptoms by modulating the gut-brain interaction in human and animal models. Neurogastroenterol Motil. 2016;28: 1027-36.

9. Messaoudi M, Violle N, Bisson JF, Desor D, Javelot $H$, Rougeot C. Beneficial psychological effects of a probiotic formulation (Lactobacillus helveticus R0052 and Bifidobacterium longum R0175) in healthy human volunteers. Gut Microbes. 2011;2: 256-61.

10. Romijn AR, Rucklidge JJ, Kuijer RG, Frampton C. A double-blind, randomized, placebo-controlled trial of Lactobacillus helveticus and Bifidobacterium longum for the symptoms of depression. Aust N Z J Psychiatry. 2017;51:810-21.

11. Takada M, Nishida K, Gondo Y, Kikuchi-Hayakawa H, Ishikawa H, Suda K, et al. Beneficial effects of Lactobacillus casei strain Shirota on academic stress-induced sleep disturbance in healthy adults: a double-blind, randomised, placebo-controlled trial. Benef Microbes. 2017;8:153-62.

12. Kawakami N, Abdulghani EA, Alonso J, Bromet EJ, Bruffaerts R, Caldas-de-Almeida JM, et al. Earlylife mental disorders and adult household income in the World Mental Health Surveys. Biol Psychiatry. 2012;72:228-37.

13. Makino S, Ikegami S, Kume A, Horiuchi H, Sasaki H, Orii N. Reducing the risk of infection in the elderly by dietary intake of yoghurt fermented with Lactobacillus delbrueckii ssp. bulgaricus OLL1073R-1. Br J Nutr. 2010;104:998-1006.

14. Morikawa Y, Kitaoka-Higashiguchi K, Tanimoto C, Hayashi M, Oketani R, Miura K, et al. A crosssectional study on the relationship of job stress with natural killer cell activity and natural killer cell subsets among healthy nurses. J Occup Health. 2005;47:378-83.

15. Kinoshita T, Maruyama K, Suyama K, Nishijima M, Akamatsu K, Jogamoto A, et al. The effects of OLL1073R-1 yogurt intake on influenza incidence and immunological markers among women healthcare workers: a randomized controlled trial. Food \& Funct. 2019;10:8129-36.

16. Doi Y, Minowa M, Uchiyama M, Okawa M, Kim K, Shibui K, et al. Psychometric assessment of subjective sleep quality using the Japanese version of the Pittsburgh Sleep Quality Index (PSQI-J) in psychiatric disordered and control subjects. Psychiatry Res. 2000;97:165-72.

17. St-Onge MP, Mikic A, Pietrolungo CE. Effects of Diet on Sleep Quality. Adv Nutr. 2016;7:938-49.

18. Fukuhara S, Suzukamo Y. Manual of the SF-8 Japanese version: Institute for Health Outcomes \& Process Evaluation research, Kyoto, Japan. 2004.

19. Revicki DA, Wood M, Wiklund I, Crawley J. Reliability and validity of the Gastrointestinal Symptom Rating Scale in patients with gastroesophageal reflux disease. Qual Life Res. 1998;7:75-83. 
20. Dimenäs E, Glise H, Hallerbäck B, Hernqvist H, Svedlund J, Wiklund I. Well-being and gastrointestinal symptoms among patients referred to endoscopy owing to suspected duodenal ulcer. Scand $\mathrm{J}$ Gastroenterol. 1995;30:1046-52.

21. Moher D, Hopewell S, Schulz KF, Montori V, Gøtzsche PC, Devereaux PJ, et al. CONSORT 2010 explanation and elaboration: Updated guidelines for reporting parallel group randomized trials. BMJ. 2010;340:c869.

22. Makino S, Hemmi J, Kano H, Kashiwagi M, Hojo K, Asami Y. Anti-fatigue effects of yogurt fermented with Lactobacillus delbureckii subsp. bulgaricus OLL1073R-1 in healthy people suffering from summer heat fatigue: a randomized, double-blind, placebo-controlled trial. Nutrients 2018;10:798.

23. Magro DO, de Oliveira LM, Bernasconi I, Ruela Mde S, Credidio L, Barcelos IK, et al. Effect of yogurt containing polydextrose, Lactobacillus acidophilus NCFM and Bifidobacterium lactis HN019: a randomized, double-blind, controlled study in chronic constipation. Nutr J. 2014;13:75.

24. Guerra PV, Lima LN, Souza TC, Mazochi V, Penna FJ, Silva AM, et al. Pediatric functional constipation treatment with Bifidobacterium-containing yogurt: a crossover, double-blind, controlled trial. World J Gastroenterol. 2011;17:3916-21.

25. Tabbers MM, Chmielewska A, Roseboom MG, Boudet C, Perrin C, Szajewska H, et al. Effect of the consumption of a fermented dairy product containing Bifidobacterium lactis DN-173 010 on constipation in childhood: a multicentre randomised controlled trial (NTRTC: 1571). BMC Pediatr. 2009;9:22.

26. Yang $Y X, H e ~ M, ~ H u ~ G$, Wei J, Pages $P$, Yang XH, et al. Effect of a fermented milk containing Bifidobacterium lactis DN-173010 on Chinese constipated women. World J Gastroenterol. 2008;14:6237-43.

27. Liu J, Du C,Wang Y, Yu Z. Anti-fatigue activities of polysaccharides extracted from Hericium erinaceus. Exp Ther Med. 2015;9:483-7.

28. Chen YM, Tsai YH, Tsai TY, Chiu YS, Wei L, Chen WC, et al. Fucoidan supplementation improves exercise performance and exhibits anti-fatigue action in mice. Nutrients 2015;7:239-52.

29. Chi A, Li H, Kang C, Guo H, Wang Y, Guo F, et al. Anti-fatigue activity of a novel polysaccharide conjugates from Ziyang green tea. Int J Biol Macromol. 2015;80:566-72.

30. Xu C, Lv J, Lo YM, Cui SW, Hu X, Fan M. Effects of oat $\beta$-glucan on endurance exercise and its antifatigue properties in trained rats. Carbohydr Polym. 2013;92:1159-65.

31. Wang J, Li S, Fan Y, Chen Y, Liu D, Cheng H, et al. Anti-fatigue activity of the water-soluble polysaccharides isolated from Panax ginseng C.A. Meyer. J. Ethnopharmacol. 2010;130:421-3.

32. an W, Li T, Lao J, Song B, Shen Y. Anti-fatigue property of Cordyceps guangdongensis and the underlying mechanisms. Pharm Biol. 2013;51:614-20.

33. Surhio MM, Wang Y, Fang S, Li J, Ye M. Anti-fatigue activity of a Lachnum polysaccharide and its carboxymethylated derivative in mice. Bioorg Med. Chem. Lett. 2017;27:4777-80.

34. Geng P, Siu KC, Wang Z, Wu JY. Antifatigue functions and mechanisms of edible and medical mushrooms. BioMed Res Int. 2017;9648496. 
35. Uemura J, Itoh T, Kaneko T, Noda K. Chemical characterization of exocellular polysaccharide from Lactobacillus delbrueckii subsp. bulgaricus OLL1073R-1. Milchwissenschaft. 1998;53:443-6.

36. Zhang L, Liu C, Li D, Zhao Y, Zhang X, Zeng X, et al. Antioxidant activity of an exopolysaccharide isolated from Lactobacillus plantarum C88. Int J Biol Macromol. 2013;54:270-5.

37. Wang K, Li W, Rui X, Chen X, Jiang M, Dong M, et al. Structural characterization and bioactivity of released exopolysaccharides from Lactobacillus plantarum 70810. Int J Biol Macromol. 2014;67:718.

38. Li W, Ji J, Chen X, Jiang M, Rui X, Dong M. Structural elucidation and antioxidant activities of exopolysaccharides from Lactobacillus helveticus MB2-1. Carbohydr Polym. 2014;102:351-9.

39. Wang J, Zhao X, Yang Y, Zhao A, Yang Z. Characterization and bioactivities of an exopolysaccharide produced by Lactobacillus plantarum YW32. Int J Biol Macromol. 2015;74:119-26.

\section{Tables}

Due to technical limitations, table 1,2,3 is only available as a download in the Supplemental Files section.

\section{Figures}




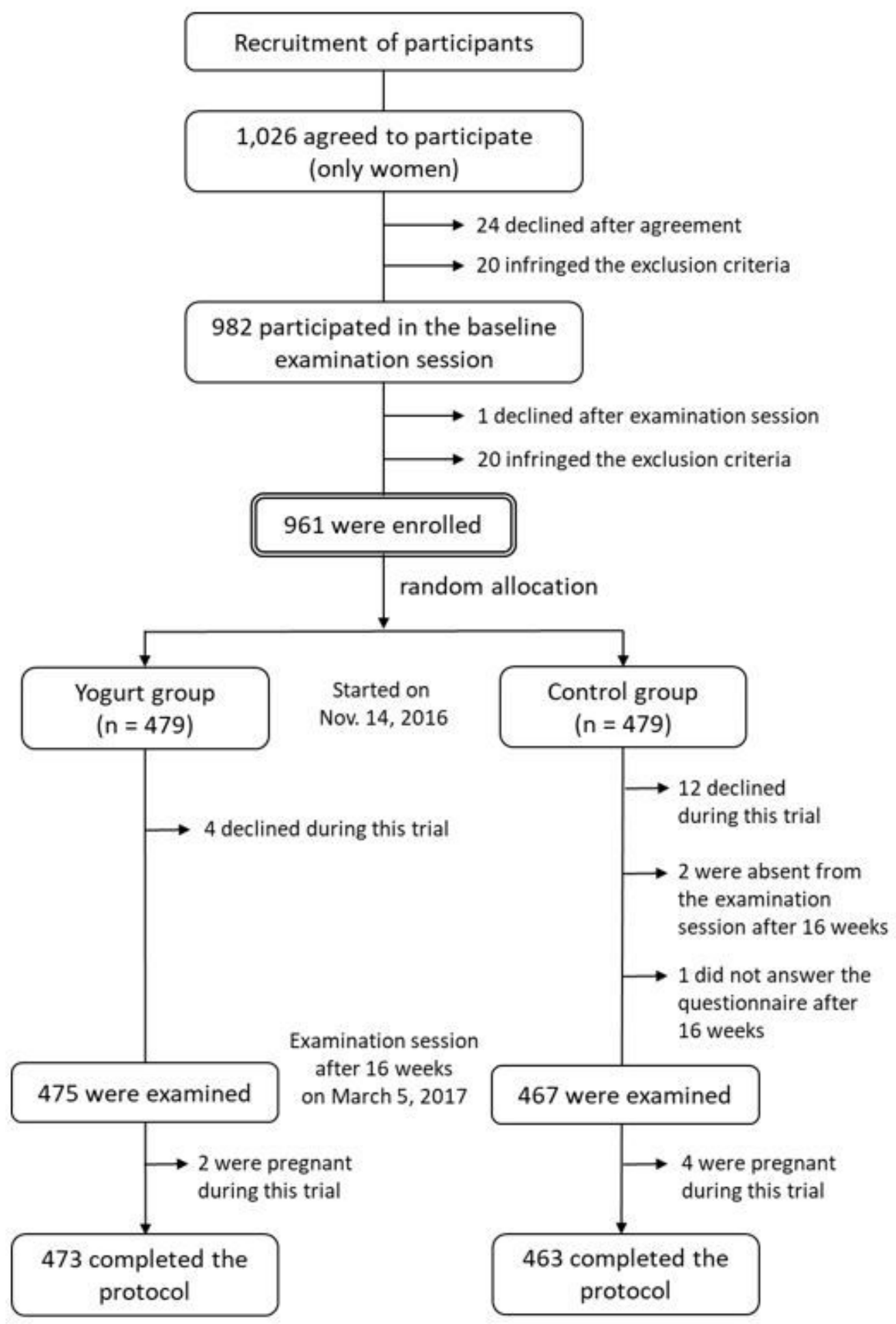

\section{Figure 1}

Sampling scheme throughout this study

\section{Supplementary Files}

This is a list of supplementary files associated with this preprint. Click to download. 
- Table1.png

- Table2.png

- Table3.png

Page 13/13 